\title{
DEVELOPMENT OF SUPERCONDUCTING QUADRUPOLES FOR HEAVY ION FUSION
}

\author{
A. Lietzke, G. Sabbi, P. Seidl, D. Shuman, LBNL, Berkeley, CA \\ C. Gung, J. Minervini, J. Schultz, L. Myatt, MIT, Cambridge, MA \\ S. Lund, B. Manahan, N. Martovetsky, LLNL, Livermore, CA \\ B. Hinson, R. Meinke, Advanced Magnet Lab, Palm Bay, FL
}

\begin{abstract}
Multi-beam heavy-ion accelerators are considered as promising drivers for inertial fusion energy. The High Current Experiment (HCX) at Lawrence Berkeley National Laboratory (LBNL) will test driver-scale beam dynamics in a single transport channel. Single bore, NbTi superconducting quadrupoles are being developed for the HCX, taking into account their possible use as modules in a quadrupole array magnet for multi-beam focusing. Two design approaches are considered, and prototype magnets of each type have been built and tested. The status of the $\mathrm{R} \& \mathrm{D}$ program is reported and a comparison of the two design options is presented.
\end{abstract}

\section{INTRODUCTION}

In a heavy-ion fusion driver, arrays of superconducting quadrupoles will transport parallel beams through a sequence of induction acceleration cells. Tight transverse packing of the beams is required to minimize the size and cost of the induction cores, leading to different optimization strategies for the magnet array with respect to conventional designs. Comparison of different approaches shows that flat windings are favoured with respect to shell-type coils. Maximizing the quadrupole gradient with minimum radial coil and structure build-up is a primary figure of merit. Field quality requirements are generally less stringent than in high-energy colliders.

The HCX is designed to explore the physics of intense beams with line-charge density $(0.20 \mu \mathrm{C} / \mathrm{m})$ and pulse duration $(3<\tau<10 \mu \mathrm{s})$ close to the values of interest for a fusion driver. A beam of $\mathrm{K}^{+}$(or $\mathrm{Cs}^{+}$) ions will be transported through a lattice of electrostatic quadrupoles followed by a 50-100 magnetic quadrupoles. Although pulsed magnets are a viable alternative for HCX, superconducting technology is the most attractive in view the ultimate fusion driver application. Initial experiments will be performed with a $1.71 \mathrm{MeV}$ coasting beam, but the design should be compatible with induction acceleration planned for later stages.

Superconducting magnet development for HCX and future machines is taking place at Lawrence Livermore National Lab (LLNL) and Advanced Magnet Lab (AML) $[1,2]$. Support with design and testing is provided by MIT Plasma Science and Fusion Center and LBNL.

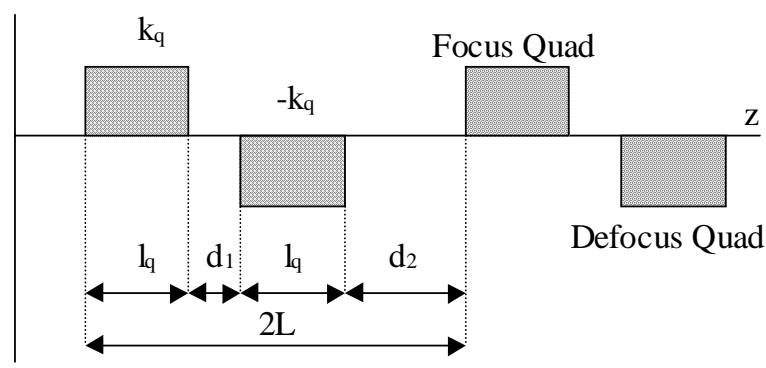

Fig. 1: HCX lattice

\section{MAGNET PARAMETERS}

Due to low beam energy, the HCX lattice has a short period of $45 \mathrm{~cm}$. At the same time, gaps are needed between cryostat tanks to allow axial space for induction acceleration, diagnostics and pumping ports, leading to a challenging packing. Lattice syncopation is used to gain sufficient axial space for cryostat terminations at the intercell gaps (Fig. 1). In the hard-edge model, a nominal quadrupole gradient of $84.2 \mathrm{~T} / \mathrm{m}$ over a magnetic length $1_{\mathrm{q}}=10.1 \mathrm{~cm}$ is required. The syncopated lattice results in a short drift with $\mathrm{d}_{1}=6.22 \mathrm{~cm}$ and a long drift with $\mathrm{d}_{2}=18.58 \mathrm{~cm}$. Fig. 2 shows how the space is allocated to the hardware in the case of one cryostat per lattice period, a configuration that maximizes the number of gaps available for acceleration and diagnostics. A physical coil length of $12.5 \mathrm{~cm}$ is specified to account for fringe field decay at the magnet ends. Additional $3 \mathrm{~cm}$ are allowed for coil mechanical support, coil to coil transitions and

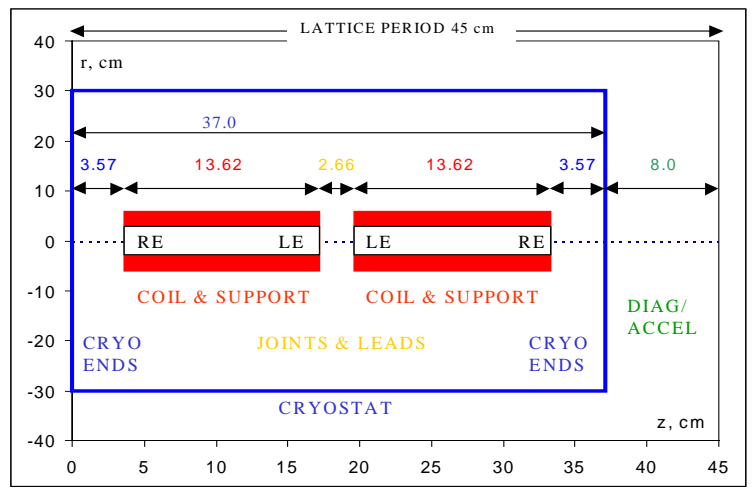

Fig. 2: Axial space allocation in each cell 

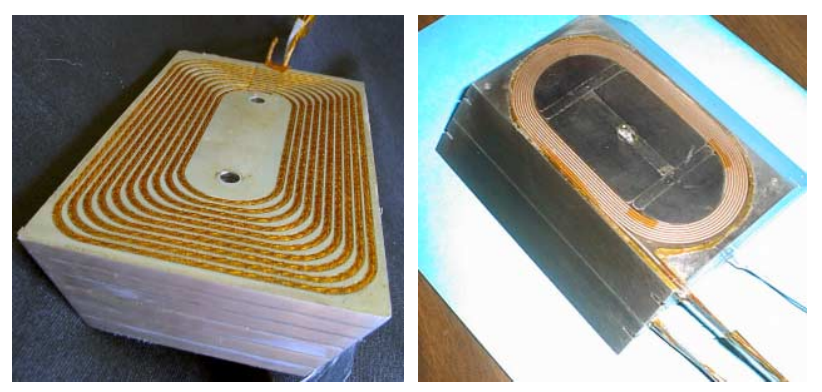

Fig. 3: AML (left) and LLNL (right) coil modules.

current leads. The short drift is used to provide electrical feeds to the magnets, with lead ends facing each other. The long drift is partially allocated to cryostat terminations. The available warm axial gap is $8 \mathrm{~cm}$.

The required magnet aperture is $70 \mathrm{~mm}$. This value applies to the structure only, without including the beam tube. The operating current $\mathrm{I}_{\mathrm{op}}$ is defined as $85 \%$ of the short sample limit current $I_{\mathrm{ss}}$. The minimum integrated gradient at $\mathrm{I}_{\mathrm{op}}$ is $8.5 \mathrm{~T}$. Magnet field quality is specified in terms of axial integrals of the 3D magnetic field components. For any longitudinal field integral calculated at $25 \mathrm{~mm}$ radius and $0<\theta<2 \pi$, a maximum deviation of $0.5 \%$ from the ideal quadrupole field at that location is allowed. The use of integrated field errors is well suited to short magnets with strong longitudinal field variations, and implicitly allows field error compensation between the magnet straight section and ends. Simulation studies of intense beams have shown that minimization of local field errors is desirable but not needed for the HCX application provided the integrated error is in the range specified [3].

\section{DESIGN AND FABRICATION}

In the AML approach, grooved plates support a round 7 -strand $(6 \times 1)$ cable. Each set of two plates forms a double pancake winding. The plates are painted with adhesive (CTD-1 PFS/1Z) and each stack of 6 plates is cured under pressure to form a monolithic subcoil (Fig. 3, left). The magnet consists of four subcoils arranged in square geometry, surrounded by iron yoke with a square aluminum frame providing preload against Lorentz forces (Fig. 4, left). Transition inserts at each plate and a special interconnection flange allow continuous winding of the entire magnet with no joints. Aluminum was initially selected as structural material for the supporting plates. However, due to turn to turn shorts developing in the coil, G-11 composite was used in the first prototype.

The LLNL double pancake coils are wound around iron cores and preloaded using stainless steel holders and keystoned wedges (Fig. 3, right). The inner and outer layer coils of each quadrant are vacuum pressure impregnated to form four monolithic sub-assemblies which are aligned at their mitered corners. Joints are used to connect the coils in series. A 4-piece iron yoke surrounds the coils and a welded stainless steel outer shell provides support against Lorentz forces (Fig. 4, right).
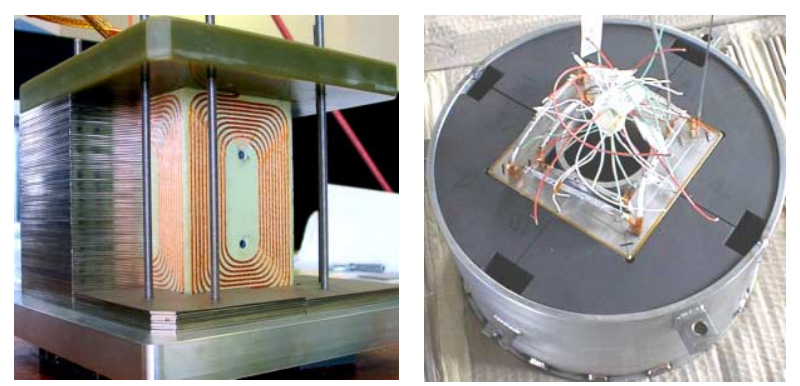

Fig. 4: AML (left) and LLNL (right) assemblies

Two prototypes have been fabricated. The first uses monolithic $\mathrm{NbTi}$ conductor manufactured with the Artificial Pinning Center (APC) process [4]. The APC conductor can achieve higher critical current density than conventional NbTi wire at fields below 5-6 $\mathrm{T}$, and has a potential for lower cost in large scale production. Although it does not present a significant advantage for HCX, it is being developed in view of its possible use in quadrupole arrays. The second model uses a conventional Rutherford cable made of 13 SSC outer strands.

Although transverse space limitations are not a key issue in the HCX, the integrated gradient that can be achieved in the bore for a given radial coil envelope is a useful figure of merit in view of future array applications. A direct comparison between the two approaches will be possible after each design is fully optimized. The AML support plates decrease the average current density with respect to conventional coils, but allow individual placement of each turn. This feature results in a good fit of the available transverse space between the elliptical beam envelope and the square cell allocated to each channel. In the end regions, the same minimum bending radius can be used for all turns (Fig. 3, left) increasing the straight section length for turns located towards the magnetic mid-plane. This results in 7\% higher integrated gradient with respect to the case of concentric turns [5]. In the LLNL design all turns in a coil have equal straight section, but spacers could be used for the same purpose.

Extending the straight section length of mid-plane turns is also beneficial from the end field quality standpoint, since it generates a positive contribution to the dodecapole which compensates the natural negative contribution from the arc. As a result, the AML design has separately optimized straight section and ends while the LLNL design uses body-end compensation to achieve low integrated harmonics. Predicted field quality for both magnets is acceptable from the beam physics standpoint.

Iron inserts at the magnetic pole can be used in single aperture quadrupoles to enhance the bore field while reducing the coil peak field. Saturation harmonics due to the magnetic inserts are not a major concern for the HCX since the magnet operates DC with a relatively small tuning range. The LLNL design incorporates iron cores as a basic feature with structural support as well as magnetic function. The AML design will incorporate iron inserts from the second prototype, resulting in a $17 \%$ increase of gradient and 7\% decrease of peak field on conductor [6]. 


\section{TEST RESULTS}

Two prototypes of the LLNL design and one prototype of the AML design have been fabricated and tested. A second AML prototype is being fabricated.

The first LLNL model has monolithic APC conductor with low copper fraction $(\mathrm{Cu}: \mathrm{Sc}=1: 1)$ and critical current density of $2.75 \mathrm{kA} / \mathrm{mm}^{2}$ ( $\left.5 \mathrm{~T}, 4.2 \mathrm{~K}\right)$. The calculated short sample current $\left(\mathrm{I}_{\mathrm{ss}}\right)$ is $3.1 \mathrm{kA}$ at $4.2 \mathrm{~K}$, corresponding to $11.2 \mathrm{~T}$ integrated gradient. The magnet was tested at LBNL in February 2001 [7]. The first quench was at $2.3 \mathrm{kA}$. From there, rapid training was observed and the magnet reached short sample after 4 more quenches. Most training quenches originated at the layer-to-layer transition of the double pancake windings. Rapid quench propagation (quench-back) was observed as well as strong ramp rate sensitivity. However, the magnet could be safely ramped to operating current at $20 \mathrm{~A} / \mathrm{s}$, which is more then adequate for HCX. The maximum joint resistance was $2.6 \mathrm{n} \Omega$. At the end of the first thermal cycle, a fault detector failure resulted in slow power supply turn-off. A second thermal cycle found no damage due to this event. No retraining was observed.

The second LLNL prototype uses a Rutherford cable with same width $(4.05 \mathrm{~mm})$ as the APC conductor but different thickness $(1.17 \mathrm{~mm}$ instead than $1 \mathrm{~mm})$ resulting in a smaller number of tuns in the coil and lower transfer function. The cable has 13 strands of the SSC outer type with critical current density similar to the APC conductor but higher copper fraction (1.8:1). For this reason the second prototype achieves a lower integrated gradient of $9.8 \mathrm{~T}$ at $\mathrm{I}_{\mathrm{ss}}=3.0 \mathrm{kA}$. A custom wire could be fabricated with smaller diameter and lower $\mathrm{Cu}: \mathrm{Sc}$ ratio than the SSC outer strand to obtain the same performance parameters as for the APC conductor. However, the focusing strength for the second model is still close to HCX specification, and higher copper fraction results in more robust operation. In fact, due to conductor packing requirements all HCX designs have high copper current density of 1.4$1.6 \mathrm{kA} / \mathrm{mm}^{2}$ at short sample. The magnet was tested at LBL in March 2001 [8]. Short sample current was reached with no training (Fig. 5). No performance degradation was observed for ramp rates up to $200 \mathrm{~A} / \mathrm{s}$.

The first AML prototype was tested at MIT in May 2001 [9]. The computed short sample current is $2.45 \mathrm{kA}$ assuming no cabling degradation. The integrated gradient at short sample is $11.3 \mathrm{~T}$, but the coils in this prototype are $2.5 \mathrm{~cm}$ longer with respect to final HCX specification. The first quench was at $1.90 \mathrm{kA}$. After 10 quenches, the magnet reached $2.20 \mathrm{kA}\left(0.90 \cdot \mathrm{I}_{\mathrm{sS}}\right)$. A second thermal cycle was performed, and further training was observed up to $2.32 \mathrm{kA}\left(0.95 \cdot \mathrm{I}_{\mathrm{ss}}\right)$. The quenches occurred in all four quadrants and did not involve the quadrant to quadrant transitions. Possible explanations for the observed behaviour are conductor movement under Lorentz forces or reduction of the current-carrying capacity of the round cable due to the central (untransposed) strand. Improvements in mechanical support as well as a

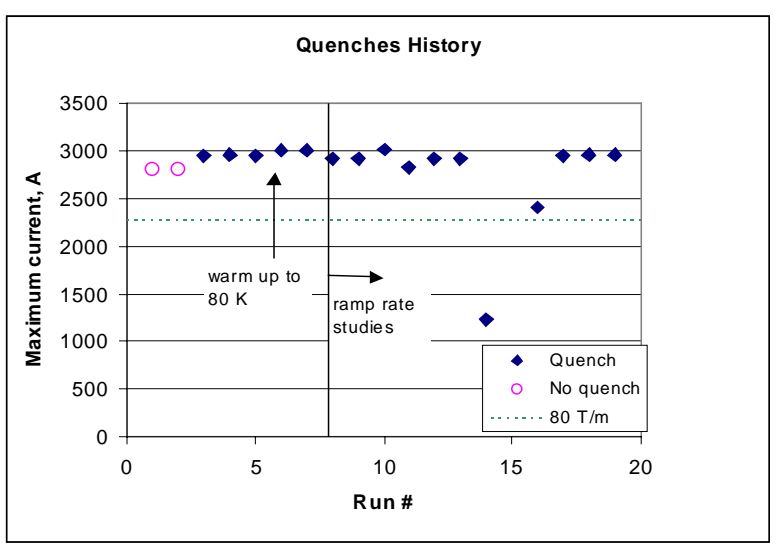

Fig. 5: Training history of second LLNL prototype [8]

modified cable design using using a central copper wire are being considered for the second prototype.

\section{SUMMARY}

Superconducting quadrupoles with 8.5 Tesla integrated gradient, $70 \mathrm{~mm}$ clear bore and $10 \mathrm{~cm}$ magnetic length are being developed for the LBNL high current experiment. Two design approaches are under study. Model magnets of each design have been built and tested showing that both are suitable for use in the HCX. Future plans include optimization of each design based on fabrication experience and test results; field quality measurements and comparison with calculated values; fabrication and test of a cryostat unit with 2 quadrupoles; and selection of the baseline HCX superconducting quadrupole design based on performance and cost of the prototype options.

\section{REFERENCES}

[1] N. Martovetsky, R. Manahan, "Focusing Magnets for HIF based on Racetracks", IEEE Trans. Appl. Supercond. Vol. 11, No. 1, March 2001, pp. 1506.

[2] R. Meinke, C. Goodzeit, W. Hinson, C. Gung, J. Minervini, A. Radovinsky, J. Schultz, B. Smith, R. Camille, L. Myatt, "Multi-Cylinder Quadrupoles with Square Cross Section", 2001 Particle Accelerator Conference, Chicago, June 2001.

[3] S. Lund, G. Sabbi, P. Seidl, "Characterization of Prototype Superconducting Magnetic Quadrupoles for the High Current Transport Experiment", LBL note HCX-MAG-01-0222-01, December 1999.

[4] T. Wong, M. Rudziak, J. Seuntjens, J. Wong, "Variable Composition NbTi Superconductors Produced by Artificial Pinning Center Process", IEEE Appl. Supercond. Vol 7, No. 2, June 1997, pp. 1126.

[5] L. Myatt, "End Turn Improvements in Optimized 6-Plate, Square X-Section Quadrupole" HCX-MAG-00-1121-01, November 2000.

[6] L. Myatt, "Field Effects from Iron Plug in AML/MIT 6-Plate Quadrupole", HCX-MAG-01-0323-01, March 2001.

[7] A. Lietzke, "HCX-APC Test Report" HCX-MAG-01-022101 and HCX-MAG-01-0228-01, February 2001.

[8] N. Martovetsky, "Results of the LLNL 5e HCX Rutherford Quadrupole Prototype", HCX-MAG-01-0329-01, March 2001.

[9] C. Gung, "HCX-AML Prototype Test Report", HCX-MAG01-0523-01, May 2001. 